\title{
On chaotic state indications of time series of failure rate of overhead lines
}

\author{
Irek Galiaskarov ${ }^{1}$, Misrikhan Misrikhanov $^{2}$, Vladimir Ryabchenko ${ }^{3}$, Andrey Shuntov ${ }^{2}$ \\ ${ }^{1}$ JSC "CEMC UES”, Moscow, Russia \\ ${ }^{2}$ National research university "MPEI", Moscow, Russia \\ ${ }^{3}$ JSC "R\&D Center FGC UES”, Moscow, Russia
}

\begin{abstract}
The accident rate of $500 \mathrm{kV}$ overhead lines (OHL) of a large region on a long time interval is researched. Significant fluctuations in the values of their failure rate (failure frequency) are revealed. The specified parameter was analyzed using the mathematical apparatus of the theory of deterministic (dynamic) chaos. The fractality of the time series of the OHL failure rate was revealed, as well as the positiveness of its maximal Lyapunov exponent, which indicated the chaotic nature of the dynamic process under consideration. The insignificant (less than five years) depth of forecasting the reliability characteristics of overhead lines due to the indicated chaotic state is substantiated. This is an unfavorable factor that reduces the reliability of the reliability estimates of the main grid of power systems.
\end{abstract}

\section{Introduction}

In [1], the cyclicity of accident rate of $500 \mathrm{kV} \mathrm{OHL}$ in a large region over an extended time interval was researched. Significant fluctuations in the values of their failure rate (failure frequency) were revealed under the influence of natural and socio-economic factors (Fig. 1); it is proposed to consider the specified parameter as the output signal of a dynamic system with many difficult to formalize inputs.

It is catching the attention that the graph in Fig. 1 (in fact - a time series) is not ordered, regular. In such conditions, the problem of predictability of accident rate in the main grids of power systems is of interest. For example, in [2], such variability in the failure frequency of a $500 \mathrm{kV}$ overhead line was described by a mathematical model containing a linear trend and a set of harmonic components determined using the Fourier transform.

It was noted that it reflects the trend of changes in the accident rate of OHL (including taking into account the cycles of solar activity) with an accuracy acceptable for predictive estimates, and the random component can be represented in the form of Gaussian white noise. However, the wavelet spectrum of the graph in Fig. 1 revealed [1] the presence in the frequency spectrum of the failure rate of three distinguishable time periods with sharply not uniform reliability pecharacteristics. Therefore, the use of these harmonic components seems to be justified within the framework of a separately taken, limited time period, i.e. without taking into account the previous historical "tails" of data.

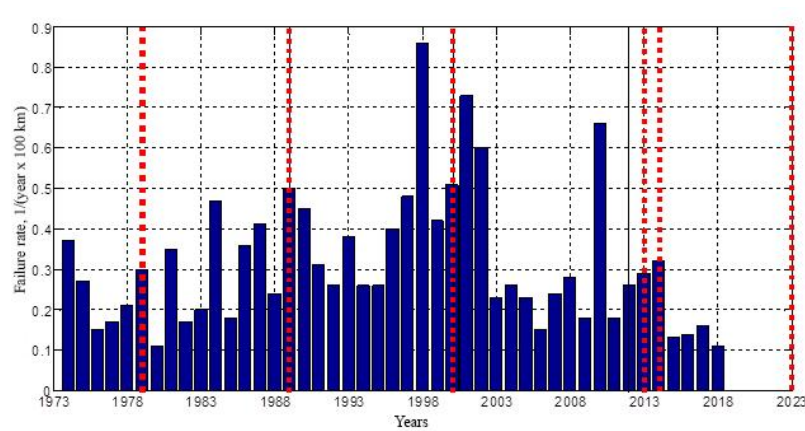

Fig. 1. Failure rate values of $500 \mathrm{kV}$ OHL for the period 1974-2018. (reported data) with overlapping solar cycles (dotted line)

Therefore, to solve the problem of predicting the accident rate of overhead lines, the authors of this paper have involved the theory of deterministic (dynamic) chaos, which is presumably more appropriate in this case (see the review in $[3,4])$. In the first approximation, the presence of fractality (or, as they say, the phenomenoncompanion of chaos) and the positivity of the maximal Lyapunov exponent (a sign of instability of a dynamical system) are used as criteria for the chaotic behavior of the dynamical system under consideration.

\section{Fractal dimension calculation}

As is well known, the statistical characteristic of chaos is the dimension of a strange attractor, which differs from the usual dimension of a point, line, plane, volume, ... (dimensions, respectively, zero, one, two, three, ...). Strange attractors have a fractal structure, and, accordingly, their dimension is fractional.

To determine the fractal dimension $D$, one of the most suitable for such (Fig. 1) time series was used the

* Corresponding author: Galiaskarov_im@center.cius-ees.ru 
Higuchi method $[5,6]$. The algorithm for calculating $D$ of the failure rate $\omega$ as a time series containing $N$ elements includes the following steps [7]:

1. Creation of new time series. From the given series $\omega$, new time series $\omega_{k}^{m}$ are created, defined as follows:

$$
\begin{gathered}
\omega_{k}^{m}: \omega(m), \omega(m+k), \omega(m+2 k), \ldots, \omega(m+ \\
\left.\left\lfloor\frac{N-m}{k}\right\rfloor k\right), m=1,2, \ldots, k,
\end{gathered}
$$

where $k$ and $m$ are integers, $m$ - the initial time, $k$ - the time interval, $\lfloor\cdot\rfloor$ - the operation of taking the whole fraction downward, for example $[2,7]=2,[2,3]=2$, $[2,01]=2$, etc.

For a time interval equal to $k, k$ sets of new time series are obtained. So for $k=3$ and $k=45$ (i.e. 45 years as in Fig. 1), the three time series obtained in this way have the following representation:

$$
\begin{aligned}
& \omega_{3}^{1}: \omega(1), \omega(4), \omega(7), \ldots, \omega(43) ; \\
& \omega_{3}^{2}: \omega(2), \omega(5), \omega(8), \ldots, \omega(44) ; \\
& \omega_{3}^{3}: \omega(3), \omega(6), \omega(9), \ldots, \omega(45) .
\end{aligned}
$$

2. Calculation of the length of each new series. The length of the curve $\omega_{k}^{m}$, denoted as $L_{m}(k)$, is defined as follows:

$$
\begin{aligned}
& L_{m}(k)=\left\{\sum_{i=1}^{\left\lfloor\frac{N-m}{k}\right\rfloor}[\omega(m+i k)\right. \\
& -\omega(m+(i-1) k)] \\
& \left.\cdot \frac{N-1}{\left\lfloor\frac{N-m}{k}\right\rfloor k}\right\}
\end{aligned}
$$

where the fraction $\frac{N-1}{\left[\left(\frac{N-m}{k}\right) k\right]}$ is the normalization factor for the length of the curve of the set of time series. The averaging $\left\langle L_{m}(k)\right\rangle$ is performed over all $m$ from 1 to $k$.

3. Construction of the graph $\left\langle L_{m}(k)\right\rangle=f(k)$. The graph of the function of the average length of the curve $\left\langle L_{m}(k)\right\rangle$ is plotted as a function of $k$ on a double logarithmic scale.

4. Determination of the fractal dimension $D$ as the modulus of the tangent of the slope of the graph $\left\langle L_{m}(k)\right\rangle=f(k)$ (it is assumed that the relation $\left\langle L_{m}(k)\right\rangle \approx k^{-D}$ is fulfilled and on this basis the dimension $D$ is estimated).

Fig. 2 shows the dependence of the values of the fractal dimension of the time series of the failure rate of the $500 \mathrm{kV}$ OHL from 1974 to 2018 years. $D_{1974-2018}$ from $k$, calculated according to items 1-4. As seen from Fig. 2, except for $k=3$, all other values of the time interval give a fractal dimension less than 2. More precisely, the averaged value for all $k$ gives $\left\langle D_{1974-2018}\right\rangle=1,8926$. Thus, from the standpoint of Euclidean geometry, we have a figure in its dimension, located between the line and the plane, but closer to the plane. Consequently, the fractality of the time series in Fig. 1 is indeed the case.

\section{Calculation of the maximal Lyapunov exponent}

To determine the characteristic exponent of the time series in Fig. 1 - the maximal exponent (first exponent) of Lyapunov $\lambda$ - the Wolf method was chosen [8]. The $\lambda$ exponent was calculated as follows. For the time series $\mathrm{x}$, a phase portrait was formed in the n-dimensional space of some dynamical system, in which any point of the series is expressed by a sequence of numbers

$$
\left\{\omega\left(t_{0}\right), \omega\left(t_{0}+\tau\right), \ldots, \omega\left(t_{0}+(n-1) \tau\right)\right\}
$$

where $\tau$ and $n$ are respectively the selected delay time and dimension of the state space of the dynamical system (embedding dimension)

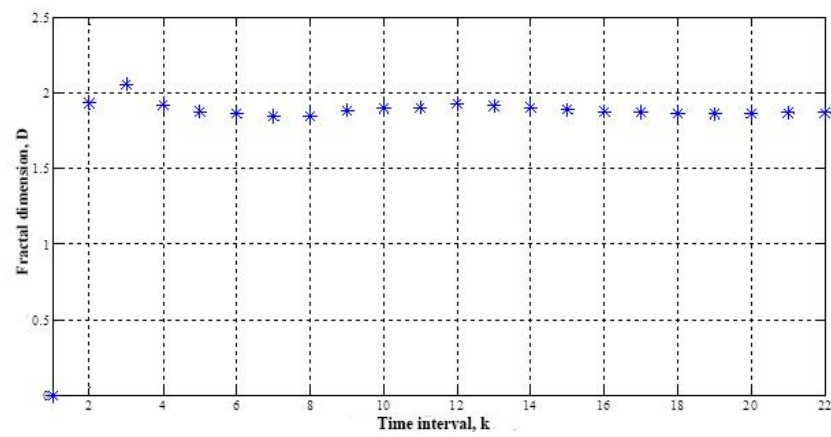

Fig. 2. $D_{1974-2018}$ of the $500 \mathrm{kV}$ OHL failure rate depending on the time interval $k$

Next, the nearest neighboring point to the starting point was determined and the distance between these two points $d\left(t_{0}\right)$ was calculated. At the next moment in time, i.e. after a given evolution time $t_{0}$, the distance between these two points became equal to $d^{\prime}\left(t_{1}\right)$. Then a new point was found that satisfies the following conditions: its distance from the displaced starting point is small in relation to the norm of the time series considered as a vector (approximately $5-7 \%$ ); the angle $\theta$, formed by the displaced start point following the sequence point (point at time $t_{1}$ ) and the selected point, is small enough (in this case, not exceeding 1-5 degrees). Then everything was repeated for the next point in the sequence.

The maximal Lyapunov exponent was calculated by the formula

$$
\lambda=\frac{1}{t_{N}-t_{0}} \sum_{k=1}^{M} \log _{2}\left(\frac{d^{\prime}\left(t_{k}\right)}{d\left(t_{k-1}\right)}\right)
$$

where $M$ is the number of the last iteration of calculating the distance between displaced points $d$ '.

There is an opinion about the difficulties of applying the Wolf method: the need to set the parameters of the method, namely, the delay time and the embedding dimension. However, effective algorithms have been developed to overcome these difficulties. One of these algorithms is based on a matrix procedure borrowed 
from symplectic geometry [9]. It consists in "packing" the time series into a matrix of the following form:

$$
\boldsymbol{W}=\left[\begin{array}{cccc}
\omega_{1} & \omega_{2} & \ldots & \omega_{r} \\
\omega_{2} & \omega_{3} & \ldots & \omega_{r+1} \\
\vdots & \vdots & \vdots & \vdots \\
\omega_{s} & \omega_{s+1} & \ldots & \omega_{L}
\end{array}\right]
$$

followed by varying the values of $s, r, L$ and calculating the parameters of the Wolf method based on the eigenvalues and vectors of the Hamiltonian matrix of the form $\operatorname{diag}(\mathrm{W}-\mathrm{WT})[10,11]$.

The performed computational experiments with the failure rate shown in Fig. 1 (reported data), showed that the optimal embedding dimension is $n=2$. In this case, the maximal Lyapunov exponent takes the value $\lambda_{1974-2018}=0,2183>0$. Its positive value indicates the unstable and chaotic nature of the analyzed time series, considered as an output parameter of a complex nonlinear dynamic system.

\section{Estimation of the predictive depth of the accident rate of $500 \mathrm{kV} \mathrm{OHL}$}

Chaotic systems represent a class of uncertainty models that differ in their properties from stochastic models. If in a deterministic model the future trajectory can be predicted for an arbitrarily long time ahead, and in a stochastic model an accurate forecast, generally speaking, is impossible even for an arbitrarily small time interval, then in a chaotic model the predictive error grows exponentially and, therefore, a forecast for a limited time ahead [3, 4].

As known, the Lyapunov exponent characterizes the exponential rate of divergence of close trajectories on the attractor. If at the initial moment of time the distance between the exponents is $\varepsilon_{0}$, then, after some time $\Delta t$, the distance between them will be equal to $\varepsilon_{1}$. In this case, obviously, $\varepsilon_{1}=\varepsilon_{0} e^{\lambda \Delta t}$, whence $\lambda=\frac{1}{\Delta t} \ln \frac{\varepsilon_{1}}{\varepsilon_{0}}$. Let us assume that $\varepsilon_{1}=\mathrm{e} \varepsilon_{0}$ (the distance between the exponents has increased e times relative to the initial one), therefore, $\lambda=\frac{1}{\Delta t} \ln e=\frac{1}{\Delta t}$. Therefore, the forecasting period in such problems is $T \approx \Delta t=1 / \lambda$. In our case, $\mathrm{T} \approx 1 / 0,2183=4,6$ years.

On the other hand, based on the irregularity and cyclicality of the time series of the $500 \mathrm{kV}$ OHL failure rate revealed in [2] for the period 1974-2000. (see § 1.3) V.A. Skopintsev put forward a hypothesis about the possible cyclicity of accident rate in power systems. He suggested that the accidence in electrical grids is oscillatory in nature with a period close to the (quasi) eleven-year solar cycle. It is known to affect the change in natural and climatic factors and social phenomena, as indicated by A.L. Chizhevsky at the beginning of the last century [15].

In [2], three mathematical models are implemented to predict the accident rate of $500 \mathrm{kV}$ OHL in time $\mathrm{t}$ (Table 1).
Table 1. Mathematical models of the time series of the failure frequency [2]

\begin{tabular}{|c|c|c|c|c|}
\hline \multirow[t]{2}{*}{ Component } & \multirow[t]{2}{*}{ Parameter } & \multicolumn{3}{|c|}{ Mathematical model versions } \\
\hline & & I & II & III \\
\hline \multirow[t]{2}{*}{$\begin{array}{l}\text { Straight- } \\
\text { line trend }\end{array}$} & $\begin{array}{l}a, 1 /(\text { year } 100 \\
\mathrm{km})\end{array}$ & 0,167 & 0,155 & 0,151 \\
\hline & \begin{tabular}{|l|}
$b, 1 /($ year 100 \\
$\mathrm{km})$
\end{tabular} & 0,012 & 0,013 & 0,013 \\
\hline \multirow[t]{10}{*}{$\begin{array}{l}\text { Harmonics } \\
\text { of cyclical } \\
\text { component }\end{array}$} & $\lambda_{l}, 1 /$ year & 12,8 & 11,06 & 11,06 \\
\hline & \begin{tabular}{|l|}
$G_{1}, \quad 1 /($ year \\
$100 \mathrm{~km})$ \\
\end{tabular} & 0,081 & 0,072 & 0,071 \\
\hline & $\lambda_{2}, 1 /$ year & - & 5,53 & 5,53 \\
\hline & \begin{tabular}{|lr}
$G_{2}, \quad 1 /($ year \\
$100 \mathrm{~km})$ \\
\end{tabular} & - & 0,032 & 0,029 \\
\hline & $\lambda_{3}, 1 /$ year & 3,9 & 3,69 & 3,69 \\
\hline & $\begin{array}{l}G_{3}, \quad 1 /(\text { year } \\
100 \mathrm{~km})\end{array}$ & 0,056 & 0,04 & 0,033 \\
\hline & $\lambda_{4}, 1 /$ year & 2,8 & 2,77 & - \\
\hline & $\begin{array}{l}G_{4}, \quad 1 /(\text { year } \\
100 \mathrm{~km})\end{array}$ & 0,087 & 0,082 & - \\
\hline & $\lambda_{5}, 1 /$ year & 2,4 & 2,21 & - \\
\hline & $\begin{array}{l}G_{5}, \quad 1 /(\text { year } \\
100 \mathrm{~km})\end{array}$ & 0,083 & 0,009 & - \\
\hline \multirow[t]{2}{*}{$\begin{array}{l}\text { Random } \\
\text { component }\end{array}$} & $\begin{array}{l}m, \quad 1 /(\text { year } \\
100 \mathrm{~km})\end{array}$ & $3,37 \cdot 10^{-17}$ & $-2,78 \cdot 10^{-17}$ & $1,13 \cdot 10^{-16}$ \\
\hline & $\begin{array}{l}\sigma, 1 /(\text { year } 100 \\
\mathrm{km})\end{array}$ & 0,07 & 0,1 & 0,06 \\
\hline
\end{tabular}

The notations used in Table 1: $a$ and $b$ - some coefficients of the linear trend $(a+b t) ; \lambda i=1 / f i$; fi is the fundamental frequency of the $\mathrm{i}$-th harmonic; $\mathrm{Gi}=\sqrt{A_{i}{ }^{2}+B_{i}{ }^{2}} ; A_{\mathrm{i}}$ and $B_{\mathrm{i}}$ - some parameters of harmonics; $m$ and $\sigma$ are parameters of Gaussian white noise (mathematical expectation and standard deviation).

The first version of the model from Table 1 consists of a linear trend and four harmonics, and the random component is represented as Gaussian white noise. Following the hypothesis of the existence of a relationship between the number of disturbances on OHL and solar activity, Table 1 gives the second option - a polyharmonic model, where the harmonic with a period of 11,06 years is taken as the fundamental (nyquist) frequency and four more harmonics with multiple periods are added. In the third version of the mathematical model, in comparison with the second version, the last two high-frequency harmonics are excluded at a constant fundamental frequency.

Fig. 3 shows the graphs obtained by the author [2] of a smoothed series of accident rate for $500 \mathrm{kV}$ OHL (curve 1), a random component (2), regression of 
accident rate for $500 \mathrm{kV}$ OHL with a prediction for 10 years (until 2011), calculated using the third mathematical forecasting model from the table 1 (3), as well as solar activity - Wolf numbers divided by 1000 (4).

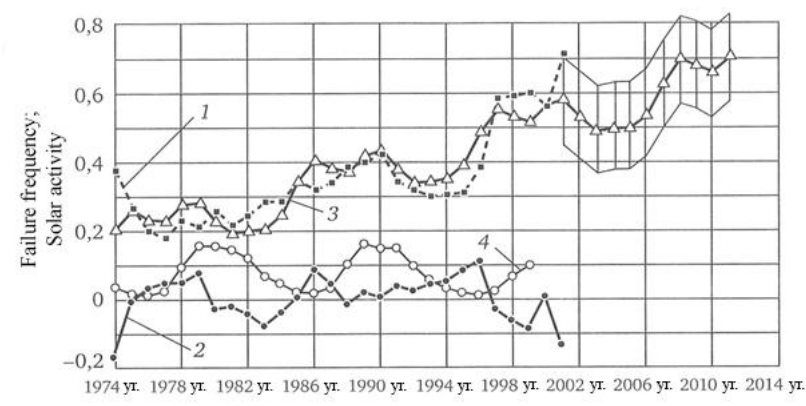

Fig. 3. Prediction of accident rate of $500 \mathrm{kV}$ OHL [2]

Research [2] in the field of predicting the accident rate of OHL should be recognized as pioneering in the past 20-year period. However, this approach to prediction appears to be extremely simplified and does not take into account the capabilities of the modern mathematical apparatus for time series prediction, which are widely used in various fields of knowledge. For example, of the traditional regression methods, there are more powerful tools - singular spectrum analysis (SSA). Here, the original series is also represented as a set of components. Only in SSA they are generally not harmonic. In addition, in recent years, prediction methods based on elements of artificial intelligence are increasingly used. And this cannot be ignored.

The above studies indicate that the time series of the failure rate is not ordered, not regular. More precisely, this series has signs of chaos expressed in fractional fractal dimension and positivity of the maximal Lyapunov exponent. It is well known that prediction technique of such series in conditions of chaotic dynamics have specific features.

Comparing the time series in Fig. 1 and the accident rate prediction in Fig. 3 in the interval 2002-2011, we note that the prediction [2] turned out to be largely unreliable. According to [2], for $500 \mathrm{kV}$ OHL in the region $\omega=0,5-0,71 /($ year $100 \mathrm{~km})$ with actual values predominantly up to $0,2-0,31 /$ (year 100$)$. The exception was the first prediction year (2002) - 0,54 1/(year 100 $\mathrm{km})$ with the fact $0,61 /($ year $100 \mathrm{~km})$, as well as the ninth prediction year (2010) - 0,67 1/(year $100 \mathrm{~km})$ with the fact $0,661 /($ year $100 \mathrm{~km})$. For the other eight years of the period under review, there were two to threefold deviations from forecast to fact. Consequently, the mathematical prediction model [2] worked only for a year ahead. Given that the maximum prediction depth using the mathematical apparatus of deterministic chaos is no more than five years.

\section{Conclusion}

The overhead lines of the main electrical grid, which form the backbone of the country's energy systems, are the most damaged elements. Therefore, their reliability should be given close attention.

The failure rate of the overhead line is traditionally considered as some variable averaged over an arbitrary time interval. Given that the accident rate of overhead lines from year to year has significant variability, irregularity and, apparently, chaos.

In the general case, the accident rate of $\mathrm{OHL}$ randomly depends on the impact of natural, social and, perhaps, socio-economic factors.

The existing approaches to predicting the accident rate of OHL in the main grids of power systems are extremely stingy and are based on the simplest regression models, which are based on the Fourier transform.

Predicting the reliability characteristics of $500 \mathrm{kV}$ OHL using the mathematical apparatus of deterministic chaos gives an acceptable forecast depth of only up to five years.

\section{References}

1. I.M. Galiaskarov, M.Sh. Misrikhanov, V.N. Ryabchenko, A.V. Shuntov, Once again about the cyclicity of accidents in the main grids of energy systems. Electrichestvo, No. 11, p. 4-11 (2019)

2. V.A. Skopintsev The quality of power systems: reliability, safety, efficiency, survivability, Energoatomizdat (2009)

3. B.R. Andrievskii, A.L. Fradkov Control of Chaos: Methods and Applications. I. Methods, Automation and Remote Control, № 64, p. 673-713 (2003).

4. B.R. Andrievskii, A.L. Fradkov Control of Chaos: Methods and Applications. II. Applications, Automation and Remote Control, № 65, p. 505-533 (2004)

5. T. Higuchi Approach to an Irregular Time Series on the Basis of Fractal Theory, Physica D. №31, p. 277283 (1988)

6. T. Higuchi Relationship between the Fractal Dimension and the Power-low Index for a Time Series: a Numerical Investigation, Physica D., №46, p. 254-264 (1990)

7. E. L. Flores-Marquez, G. Galvez-Coyt, G. CifuentesNava Fractal dimension analysis of the magnetic time series associated with the volcanic activity of Popocatepetl, Nonlinear Processes Geophysics, №19, p. 693-701 (2012)

8. A. Wolf Determining Lyapunov Exponent Form a Time Series. Physica D., №16, p. 285-317 (1986)

9. M. Lei, Z. Wang, Z. Feng A method of embedding dimension estimation based on symplectic geometry, Physics Letters, №A303, p. 179-189 (2002)

10. V.V. Voevodin, Yu. A. Kuznetsov Matrices and calculations, Nauka Fizmatlit (1984)

11. M.Sh. Misrikhanov, V.N. Ryabchenko, The quadratic eigenvalue problem in power industry systems, Automation and Remote Control, № 67, p. 698-720 (2006)

12. A. Yu., Loskutov, A.S. Mikhailov, Fundamentals of the theory of complex systems. Izhevsk Institute of Computer Science (2007) 
13. D.L. Danilov Major Time Series Components: «Caterpillar» Method, Presskom publishing house «Presscom» (1997)

14. L.N. Leontyeva, Multidimensional caterpillar, choice of length and number of components, Journal of Machine Learning and Data Analysis, №1, p. 2-10 (2011)

15. A.L. Chizhevskiy, Yu. G. Shishina, To the rhythm of the Sun, Nauka (1969) 